\title{
Repositorios y Plan S: la importancia de los secundarios en el reparto
}

Ernest Abadal | Facultat d'Informació i Mitjans Audiovisuals, Centre de Recerca en Informació, Comunicació i Cultura (CRICC), Universitat de Barcelona

URL de la contribución <www.iaph.es/revistaph/index.php/revistaph/article/view/4668>

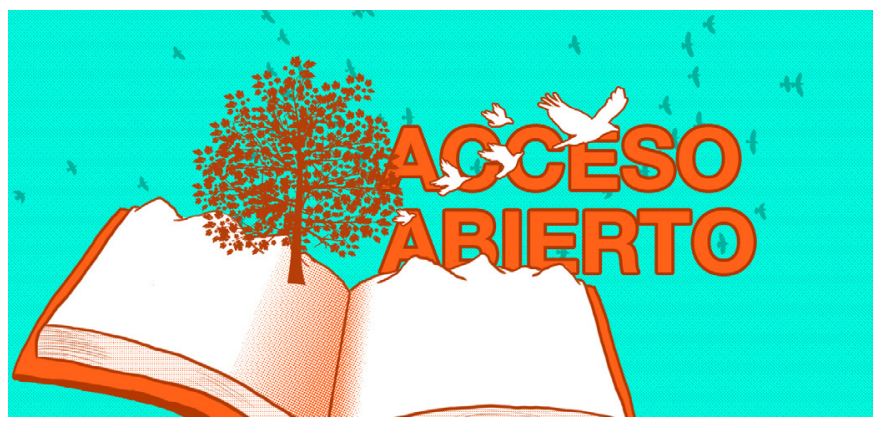

Acceso al conocimiento: la gran alternativa | imagen Fundación Karisma Colombia

Los repositorios nacieron con la etiqueta de segunda opción: la vía verde es, de hecho, un camino complementario y transitorio hacia el acceso abierto ya que cuando todas las revistas (vía dorada) sean abiertas parece que podrá prescindirse de los repositorios. De todas formas, los repositorios han reclamado protagonismo incluyendo todo tipo de documento (tesis, comunicaciones en congresos, conferencias, recursos docentes, etc.) y también incorporando nuevas funcionalidades (estadísticas, altmétricas, preservación, etc.) que les ha otorgado un alto posicionamiento en buscadores. Aunque no disponen de la facilidad y la agilidad para incorporar los contenidos o de una interfaz agradable e intuitiva como las que tienen las redes académicas, siguen siendo un elemento muy importante no solo del movimiento por el acceso abierto sino también del sistema de comunicación científica.

Este estigma de segundón, de artista secundario por detrás de las revistas, también quedó patente en el proceso de difusión del Plan S. La primera versión del plan (septiembre 2018) casi no mencionaba a los repositorios (aunque con buena voluntad se podía entender que sí se tenían en cuenta) y hubo que esperar a la segunda ver- sión (mayo 2019) para que se tuvieran en consideración, apareciendo explícitamente mencionados como una de las tres vías de cumplimiento del Plan S.

\section{¿Qué es el Plan S?}

Se trata de una iniciativa de cOAlition S (PLAN, 2019), del Consejo Europeo de Investigación (ERC) y de varias agencias estatales europeas para acelerar la transición hacia el acceso abierto. Su objetivo es conseguir que todas las publicaciones científicas derivadas de proyectos con financiación pública se publiquen en acceso abierto de manera inmediata a partir de 2021 (ABADAL; LÓPEZ-BORRULL; OLLÉ-CASTELLÀ, et ál., 2019).

Para cumplir con este objetivo, el Plan S propone tres rutas posibles a los investigadores:

> La publicación del artículo en revistas o plataformas de acceso abierto.

$>$ El depósito en repositorios (para artículos en revistas de suscripción).

> La publicación en revistas de suscripción en transición (deben tener un plan de transición al modelo de acceso abierto y finalizarlo en 2024).

La consideración de la vía verde (los repositorios) constituye un cambio notable respecto de la primera versión del plan y es un indicativo de su importancia para difundir y preservar los resultados de la investigación.

\section{¿Qué requisitos deben cumplir los repositorios?}

El Plan S incluye un conjunto de principios generales que afectan tanto a las revistas (LÓPEZ-BORRULL; OLLÉCASTELLÀ; GARCÍA-GRIMAU et ál., 2020) como también a los repositorios y que suponen un cambio notable 

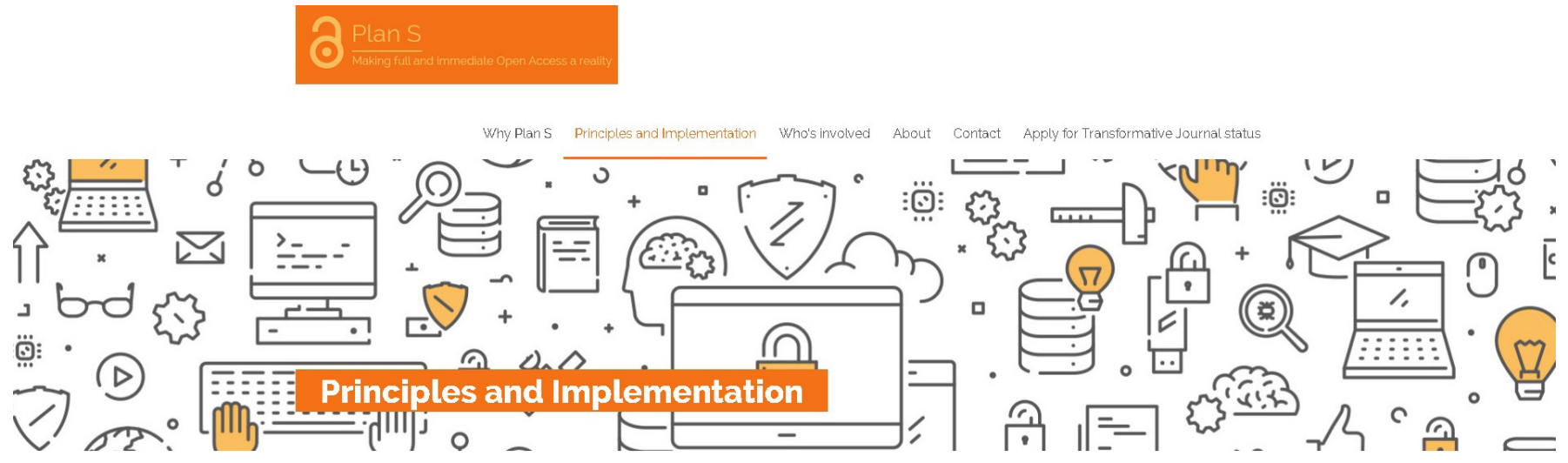

respecto de la situación actual. Vamos a comentarlos brevemente.

a) Debe archivarse la versión publicada o aceptada. Los autores podrán publicar en aquellas revistas de suscripción que permitan depositar la versión publicada (Version of Record, VoR) o la versión revisada (Author's Accepted Manuscript, AAM) en un repositorio abierto. Esta petición puede suponer un desafío a algunos repositorios por cuanto va a conllevar el depósito de distintos tipos de versiones (anteriores al Plan S y posteriores), pero también la necesidad legal de seguir controlando si los autores disponen o no de los derechos para depositar la versión aceptada.

b) Inmediatamente.

El depósito debe realizarse de manera "inmediata", sin periodos de embargo. Se pretende así acabar con una visión de preprint de contenido diferente al publicado en la revista.

\section{c) Licencias cc-by.}

Estas licencias permiten no tan sólo la libre reproducción y distribución de los contenidos, sino que también posibilitan la reutilización (traducciones y adaptaciones, especialmente) y la posible comercialización (ya sea mediante la venta de versiones impresas o en otras formas). Se trata de un tipo de licencia que hasta ahora ha tenido un papel minoritario en la difusión de con- tenidos académicos y poco utilizada, por tanto, en los repositorios.

d) También se debe archivar en repositorios.

Finalmente, de manera independiente a la vía utilizada (revista de acceso abierto o de suscripción en transición), se anima a los autores a depositar también sus artículos en un repositorio. Se indica además que algunas agencias de financiación consideran que este depósito debe ser obligatorio. Así pues, ya sea recomendación u obligación, queda claro la alta valoración que tienen las funciones de preservación y difusión de los repositorios.

Por otro lado, el Plan S dispone también de un documento (Technical Guidance and Requirements) que describe los requisitos técnicos -pero también legales y de modelo de negocio-- que deben cumplir las revistas, plataformas y repositorios para ajustarse al modelo establecido. Aquí se encuentra el apartado "Requirements for Open Access Repositories" (REQUIREMENTS, 2019) en el cual se encuentran requisitos obligatorios y otros especialmente recomendados.

A continuación, indicamos los elementos más destacados de una lista amplia a la que se debe prestar especial atención:

$>$ Estar registrado en DOAR (Directory of Open Access Repositories). 
$>$ Utilizar identificadores para las versiones de los documento (DOI, URN, Handle, etc.) y para los autores (ORCID, etc.).

$>$ Asegurar total interoperabilidad

$>$ Disponer de metadatos de alta calidad a nivel de artículo.

> Usar formatos estándares no propietarios.

$>$ Facilitar la lectura por máquina.

$>$ Asegurar un acceso ininterrumpido.

$>$ Dar servicio de atención (asegurar respuesta en un máximo de 24 horas).

> Acceso abierto a las citas siguiendo el estándar Initiative for Open Citations (I4OC).

$>$ Disponer de pasarelas con otros sistemas como PubMed, Crossref, o SCOPUS.

\section{¿Qué harán las revistas?}

La gran duda en estos momentos se encuentra en la receptividad que tendrán las revistas de suscripción a las exigencias del Plan S (en especial, a que los autores puedan depositar de manera inmediata la versión revisada y con licencia cc-by). Muchos dudan que vayan a permitirlo. Habrá que verlo. En cualquier caso, el grupo editorial Lancet ya ha aceptado el reto y permite a sus autores archivar en un repositorio la versión revisada sin ningún coste y con licencia cc-by (THE LANCET GROUP, 2019).

\section{El futuro de los repositorios}

Los repositorios deberán conocer con detalle y adaptarse a las exigencias del Plan S - una iniciativa que esperamos que sea secundada por muchas más agencias de financiación- y a otras que puedan venir. Es destacable que se hayan consolidado como una de las vías de cumplimiento del plan y, especialmente, que las agencias de financiación, como se ha comentado, recomienden y obliguen que los artículos publicados en revistas de acceso abierto también se archiven en repositorios. De esta manera, unas plataformas que nacieron como vía secundaria ven reafirmada y valorada su función y se aseguran un papel relevante en el ecosistema de la comunicación científica.

\section{BIBLIOGRAFÍA}

- ABADAL, E.; LÓPEZ-BORRULL, A.; OLLÉ-CASTELLÀ, C.; GARCIA-GRIMAU, F. (2019) El Plan S para acelerar el acceso abierto: contexto, retos y debate generado. Hipertext.net [en línea], n. ${ }^{\circ}$ 19, 2019, pp. 75-83 <https://www.raco.cat/index. php/Hipertext/article/view/360210> [Consulta: 11/05/2020] DOI:10.31009/hipertext.net.2019.i19.06

- THE LANCET GROUP (2019) Plan S: the final cut. The Lancet [en línea], vol. 393, n. ${ }^{\circ}$ 10188, $2019<$ https://doi. org/10.1016/S0140-6736(19)31322-4> [Consulta: 11/05/2020]

- LÓPEZ-BORRULL, A.; OLLÉ-CASTELLÀ, C.; GARCÍAGRIMAU, F.; ABADAL, E. (2020) Plan S y ecosistema de revistas españolas de ciencias sociales hacia el acceso abierto: amenazas y oportunidades. El profesional de la información [en línea], vol. 29, n. ${ }^{\circ}$ 2, 2020 e290214. <https://doi.org/10.3145/ epi.2020.mar.14> [Consulta: 11/05/2020]

- PLAN S. Making full and immediate open access a reality (2020) [en línea]. Strasbourg: cOAlition S, European Science Foundation, 2020 <https://www.coalition-s.org> [Consulta: 11/05/2020]

- REQUIREMENTS for Open Access Repositories (2020) En Plan S. Making full and immediate open access a reality [en línea]. Strasbourg: cOAlition S, European Science Foundation, $2020<$ https://www.coalition-s.org/addendum-to-the-coalitions-guidance-on-the-implementation-of-plan-s/principles-andimplementation/> [Consulta: 11/05/2020] 